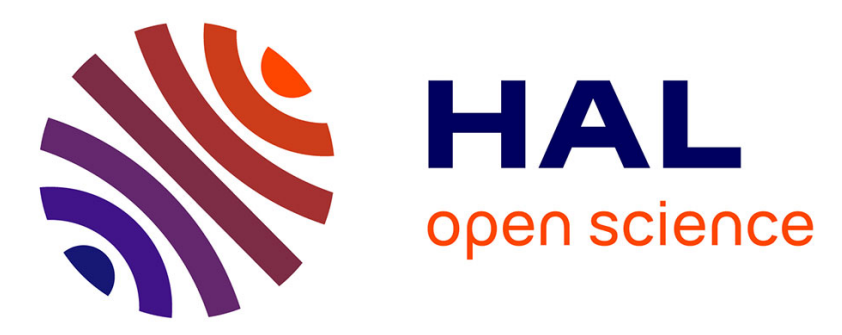

\title{
A pilot study on the dynamics of online risk assessment by the passenger of a self-driving car among pedestrians Jeffery Petit, Camilo Charron, Franck Mars
}

\section{To cite this version:}

Jeffery Petit, Camilo Charron, Franck Mars. A pilot study on the dynamics of online risk assessment by the passenger of a self-driving car among pedestrians. 22nd International Conference on HumanComputer Interaction, Jul 2020, Copenhagen, Denmark. pp.101-113, 10.1007/978-3-030-50523-3_8 . hal-02487213

\section{HAL Id: hal-02487213 \\ https://hal.science/hal-02487213}

Submitted on 3 Sep 2020

HAL is a multi-disciplinary open access archive for the deposit and dissemination of scientific research documents, whether they are published or not. The documents may come from teaching and research institutions in France or abroad, or from public or private research centers.
L'archive ouverte pluridisciplinaire HAL, est destinée au dépôt et à la diffusion de documents scientifiques de niveau recherche, publiés ou non, émanant des établissements d'enseignement et de recherche français ou étrangers, des laboratoires publics ou privés. 
Petit J., Charron C., Mars F. (2020) A Pilot Study on the Dynamics of Online Risk Assessment by the Passenger of a Selfdriving Car Among Pedestrians. In: Krömker H. (eds) $\mathrm{HCl}$ in Mobility, Transport, and Automotive Systems. Automated Driving and In-Vehicle Experience Design. HCII 2020. Lecture Notes in Computer Science, vol 12212, pp 101-113. Springer, Cham.

https://doi.org/10.1007/978-3-030-50523-3_8

\title{
A pilot study on the dynamics of online risk assessment by the passenger of a self-driving car among pedestrians
}

\author{
Jeffery Petit ${ }^{1 凶}$, Camilo Charron $^{12 \bowtie}$, Franck Mars $^{1 凶}$ \\ ${ }^{1}$ Université de Nantes, Centrale Nantes, CNRS, LS2N, F-44000 Nantes, France \\ \{jeffery.petit, camilo.charron, franck.mars\}@ls2n.fr \\ ${ }^{2}$ Université de Rennes 2, France
}

\begin{abstract}
In autonomous cars, the automation systems assume complete operational control. In this situation, it is essential that passengers always feel comfortable with the vehicle's decisions. In this project, we are specifically interested in risk assessment by the passenger of an autonomous car navigating among pedestrians in a shared space. A driving simulator experiment was conducted with 27 participants. The challenge was twofold: on the one hand, to find a link between the pedestrians' avoidance behavior of the vehicle and the risk felt by the passenger; and on the other hand, to try to predict this perceived risk in real time. The study revealed a significant effect of two factors on the risk assessed by the participants: (1) the value of the TTC at the moment the vehicle begins a pedestrian avoidance maneuver; (2) the lateral distance it leaves to the pedestrian. The proposed real-time prediction model is based on the principle of impulse response operation. This new paradigm assumes that the passenger's risk assessment is the result of a quantifiable unconscious internal phenomenon that has been estimated using the dynamics of the perceived pedestrian approach. The results showed that this approach was predictive of risk for isolated avoidance maneuvers, but was insufficient to explain the variability in the risk assessment behavior of the participants.
\end{abstract}

Keywords: Online risk assessment, driving simulator

\section{Introduction}

The advent of driver automation systems in vehicles is changing the driving paradigm [1]. At least in the near future, experts in the field consider that the driver will have to learn to cooperate with the vehicle [2]. This new role in driving suggests that the user of a vehicle equipped with an autonomous driving system will have to continue to assess his or her environment and the behavior of the car. On the one hand, this will enable them to understand the environment in which they are operating, and on the other hand, it will enable them to update their knowledge of the functional state of their car. In the introduction to his paper on the importance of taking into account processing and action times when studying human-machine interaction, Hollnagel [3] suggested that the Human and the machine have a representation of how the other works. Man develops such a representation through experience, the machine possess- 
es this representation in its design, and it is somehow transmitted by the engineers who developed it. For the driver, this representation (called "internal model") is very important because it contains all his or her knowledge on the driving behavior of the automaton. This internal model allows the driver to understand and anticipate the maneuvers of his or her vehicle. If everything goes well, i.e. if the driver feels that the driving automaton is making the right choices, the movement does not alter his emotional state (e.g. stress, anxiety) and the driver remains in a comfortable situation.

It has been shown that individuals do not necessarily change their social rules when acting with an automated system [4]. In a mixed dynamic context, where the autonomous vehicle has to make its way among other users (pedestrians, cyclists, other autonomous or non-autonomous vehicles), this may mean that the driver expects his or her vehicle to act according to the same social rules as he or she does, i.e. to adopt the same driving style as he or she does [5]. The maneuvering choices followed by the automaton in order to make its way among other users must therefore be close to the possible options considered by the driver. Gibson and Crooks [6] suggest that there is a dynamic zone that the driver perceives consciously or unconsciously in which travel can take place safely (Field of Safe Travel). The acceptable trajectory envelope that the driver perceives is subjective and depends, among other things, on the driver's experience, the safe distances he or she wishes to travel and the driver's perception of his or her own (in our case, the car's) size. It is suggested here that the trajectory perceived by the driver plays a major role in how he or she feels when driving a vehicle equipped with an autonomous driving system: the more the vehicle respects this trajectory, the better the driver will feel.

These considerations raise the question of the perceived comfort or risk on board an autonomous vehicle during a travel among other road users. Unfortunately, there are very few studies in the literature on passenger risk perception in an autonomous vehicle (e.g., [7-9]). We propose here to study this phenomenon in a dynamic context using a driving simulator. Passengers will assess their perceived risk of collision in real-time during a trip among pedestrians. The main challenge is to understand the dynamics of how a passenger feels when he or she is not in control of the vehicle in which he or she is travelling. Such an understanding could then be used to adjust the safety margins respected by automated driving cars when travelling among multiple other road users.

This paper therefore presents a pilot study of the online risk assessment in a driver simulator. It introduces a new method for the real-time study and prediction of perceived risk. In Section 2, information about the experiment and the data analysis are given. Results and interpretations are detailed in Section 3. Finally, a discussion of these results and the corresponding conclusions are presented in Section 4.

\section{Method}

\subsection{Participants}

For this experiment 27 volunteer participants were recruited aged between 18 to 52 years $(S D=7.75)$. They were 17 males and 10 females. The choice was made to 
select participants with varying degrees of driving experience. 22 participants out of 27 had a driver's license (average duration $=10$ years, $S D=8.61$ ). 2 individuals had a driver license but reported they never drive.

\subsection{Experimental setup and dependent variable}

During the experiment, participants were asked to take place in a fixed-based driving simulator (Fig. 1.a) operated with SCANeR ${ }^{\mathrm{TM}}$ Studio software [10]. They were installed in the seat of a virtual autonomous car and were asked to evaluate the risk of collision with pedestrians during a driving simulation in a shared space. Such space is intended to eliminate any segregation between road users (e.g., absence of road signs and road markings) and therefore makes any notion of priority or speed limits implicit. This urban design has already been introduced in large cities (e.g., Exhibition Road in the museum district of South Kensington in London, UK) and should, among other things, enable drivers to integrate better into their environment, resulting in lower vehicle speeds and smoother traffic flow [11-13]. During the simulation, participants were informed that the vehicle was fully automated and that no action on the controls would be required. In order for participants to assess the perceived risk throughout the simulation, an analog device has been developed for one-handed use (Fig 1.b). The objective was to avoid visual distraction; therefore, the device was designed to be used without requiring participants to look at it.

(a)

(b)

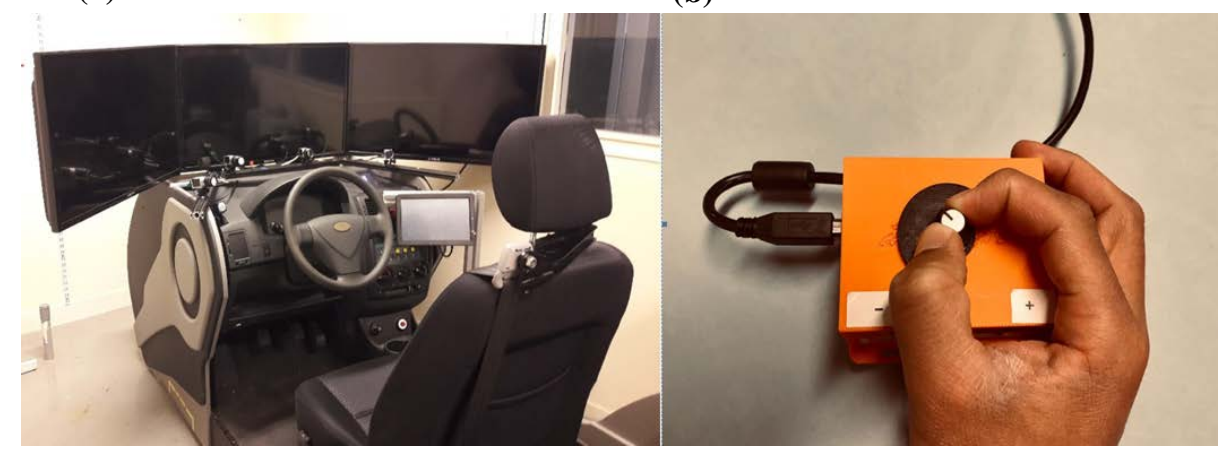

Fig. 1. Setup used in the experiment. (a) The fixed-base driver simulator operated by SCAN$\mathrm{eR}^{\mathrm{TM}}$ Studio. (b) The Analog device used for the online risk assessment. The orange box contains a potentiometer linked to an USB alimented Arduino $\mathrm{UNO}^{\mathrm{TM}}$ electronic board.

On the basis of the measures collected, 5 participants were found to be nonresponsive (they reported little risk) and have been excluded from the data analysis. 4 of them were among the 7 participants who do not have a driver license or who never drive. This may reflect the fact that driving experience may condition the attitude of passengers when they are in a self-driving car. 


\subsection{Experiment}

Procedure and instructions. As mentioned above, participants were to pay attention to the driving scene and as little as possible on the online rating operation. For this reason, prior to the main experiment, each participant conducted a preliminary scenario of autonomous driving on a pedestrian-free road; the objective for participants was to optimize the use of the device by finding a good hand position and exploring the available rating scale.

Then, in order to study the online risk assessment, there were two successive simulations, each lasting around 7 minutes of autonomous driving. The experiment has been divided into two parts with a break in between to reduce the monotony of the task. For both parts, participants were asked to use the analog device for assessing their risk of collision in real time. In both scenarios, the autonomous vehicle was travelling at a constant speed of $30 \mathrm{~km} / \mathrm{h}$. It followed a straight-line trajectory except when it had to avoid pedestrians, which happened every 25 seconds.

Independent variables. Avoidance conditions have been varied as a function of four variables:

- The time-to-collision (TTC) when the avoidance maneuver was triggered. In straight line trajectory, the TTC is the time remaining before the vehicle reaches an obstacle. It depends on both the distance and the relative speed between the vehicle and the obstacle (Fig. 2). In the experiment, 4 values of TTC were tested: 2, 2.5, 3 and 3.5 seconds.

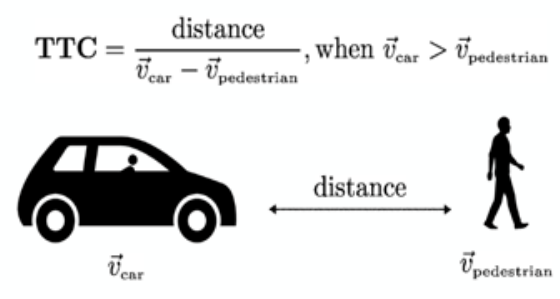

Fig. 2. The Time-To-Collision (TTC) in a straight forward trajectory. The speed of the car (resp. pedestrian) is denoted $\vec{v}_{\text {car }}$ (resp. $\vec{v}_{\text {pedestrian }}$ ). On this illustration, the pedestrian walks in the same direction as the vehicle so that the condition $\vec{v}_{c a r}>\vec{v}_{\text {pedestrian }}$ is required. This condition is respected by default when the pedestrian walks in the opposite direction $\left(\vec{v}_{\text {pedestrian }}<0\right)$.

- The lateral distance from the pedestrian when the vehicle has reached its position. This parameter was introduced to check whether the proximity between vehicle and pedestrian affects the risk assessment. 3 values of lateral distance have been tested: $0.5,1,1.5$ meters. 
The combination of the manipulation of the TTC and the lateral distance allowed simulating different safety margins. Because of software constraints, it was not possible to cross all degrees of the two variables. Indeed, with a TTC of 2 seconds it was impossible to respect a lateral distance smaller than 1.5 meter. As a consequence the experience plan was not complete: only 8 conditions out of 12 have been tested.

Two additional factors have been manipulated to make the simulations more realistic and unpredictable.

- Half of the pedestrians walked in the same direction as the vehicle (the participant could see their back), the other half walked in the opposite way (they were facing the vehicle).

- In a shared space there is no rule concerning the direction the vehicle should avoid other road users. For that reason, the direction of the avoidance maneuver was varied between left and right.

In addition to the 4 parameters influencing avoidance conditions, the pedestrians' appearance was arbitrarily chosen from a list of a dozen possibilities (man in T-shirt, man in suit, teenager in shorts, etc.).

\subsection{Data Analysis}

Factor analysis. One of the objectives of this study was to find out whether participants' risk assessment was dependent on pedestrian avoidance conditions. An avoidance maneuver is supposed to create a reaction from the participant in terms of the assessed risk. More concretely, an evolution of the assessed risk is expected for each pedestrian avoided. As mentioned before, the pedestrians were very far apart from each other. The advantage of such a design is twofold: on the one hand, it guarantees that the risk assessment necessarily concerns one and only one pedestrian, on the other hand, it guarantees that the risk assessment starts and ends at 0 . Hence, for each avoided pedestrian, a time series of assessed risk was extracted. Data were primarily scaled to be in the range $[0 ; 1]$, and then two indicators were computed: the maximum assessed risk (Risk Max) and the area under the risk curve (AUC). Those indicators are illustrated on Figure 3. It resulted in two sets of 32 indicator values by participant for the two experimental phases. The influence of the independent variables (TTC, lateral offset, pedestrian heading and the direction of the avoiding maneuver) on those values was assessed by means of analyses of variances. 


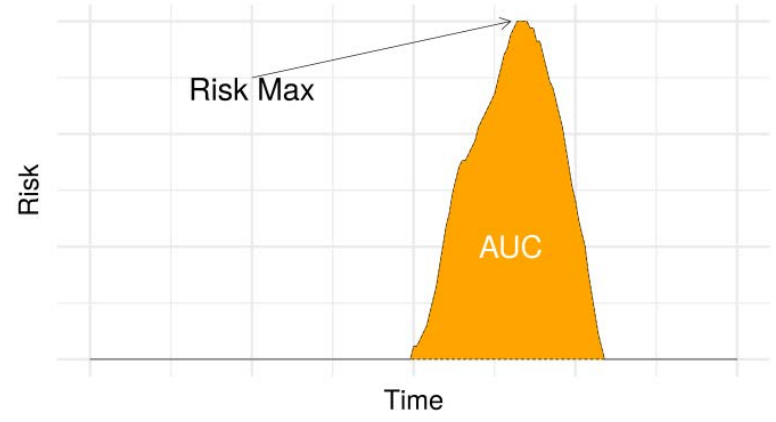

Fig. 3. Example of the assessed risk by a participant for a maneuver of pedestrian avoidance. Two indicators were computed: the maximum assessed risk (Risk Max) and the area under the risk curve (AUC). It is important to mention that the unimodal specificity of the curve was common to all participants.

Dynamics analysis of the assessed risk. In the considered approach, risk assessment is the external expression of an implicit internal phenomenon that can be modelled as an impulse response (IR). Formally, an IR is a dynamic function that gives the quantitative reaction of a system to a unitary stimulus (a Dirac impulse). In this context of online risk assessment, the objective of the IR is to represent the dynamics of the cognitive process that leads to the participant's rating when the vehicle avoids a pedestrian. Consequently, the quantitative data collected for each participant should be considered as time series of the risk assessment that is assumed to be the response to a certain stimulus flow.

Such a paradigm assumes that risk assessment can be seen as the response of a linear and time-invariant system to a given input signal [14]. Linearity requires that the risk assessment (output) in the case of a response to a linear combination of stimuli (inputs) should be the same linear combination of the output responses of the individual inputs. A simple way to illustrate this concept is to say that the response to a 10 unit stimulus should be 10 times the response to a 1 unit stimulus whatever the unit. The time invariance implies that the response to a stimulus should be independent to the moment the stimulus is perceived. That is, a stimulus occurring at a time $t$ should produce the same reaction as the same stimulus occurring at a time $t+\Delta$ whatever is the $\Delta$.

The IR is computed using an autoregressive model with exogenous variable (called an ARX model). Given a time $t$, a general formula is given in the equation 1 . The value of risk assessment (the output, denoted Risk) is supposed to depend on the $n a$ previous values of risk assessment, on $n b$ values of an exogenous variable (the input, denoted $X$ ) with a delay of $n k$ time units and on a white noise disturbance (denoted $\varepsilon$ ) mainly attributed to measurement error or to uncontrollable inputs phenomena.

$$
\operatorname{Risk}(t)=\sum_{i=1}^{n a} \alpha_{i} \times \operatorname{Risk}(t-i)+\sum_{j=0}^{n b-1} \beta_{j} \times X(t-n k-j)+\varepsilon(t)
$$

As seen in the equation 1, an ARX model is completely defined with 3 parameters: 
- $n a$ : Number of autoregressive components, also called the output samples.

- $n b$ : Number of exogenous components, also called the input samples.

- $n k$ : Number of input samples (exogenous) that occur before the input affects the output, also called the dead time in the system.

Those parameters have to be rigorously chosen as they directly condition the form of the IR. The latter can be seen as a time series where each value is a linear combination between, on one side the coefficients $\alpha_{i}$ and the output series and on the other side the coefficients $\beta_{i}$ and the input series. In this experimental case, the optimal numbers of coefficients (i.e. $n a$ and $n b$ ) were to be found using the data. A grid search was performed to find out the best configuration (performance test among combinations of the parameters).

In this study, the exogenous variable introduced in the ARX model is the evolution of the retinal expansion rate of the pedestrian. If we consider two points belonging to the pedestrian, the retinal expansion rate is the dilation rate of the optical angle (denoted $\phi$ ) formed by those two points as illustrated on Fig. 3. With some trigonometric approximations, the rate of retinal expansion (i.e. $\phi / \dot{\phi}$ ) has been shown to be the inverse of TTC [15].The use of this variable in this study of online risk assessment turns out to be consistent with many references in the literature about the detection and avoidance of upcoming collision by drivers [16-18].

$$
T T C=\frac{\phi}{\dot{\phi}}
$$

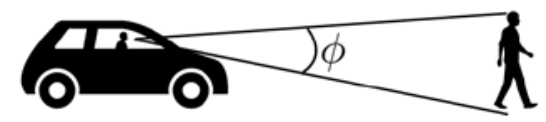

Fig. 4. As demonstrated by Lee [15], with some trigonometric approximation the TTC can be expressed as the inverse of the rate of dilatation of the optical angle represented by (any) two points on the pedestrian.

\section{Results}

\subsection{Effect of TTC and lateral Offset}

As explained before, two indicators (Risk Max and AUC) have been computed for each avoided pedestrian and analysis of the variance (ANOVA) were performed to figure out whether the factors affected the observed risk assessment. To do that, each factor was studied independently. A preliminary analysis revealed that neither the direction of the avoidance maneuver (left/right) nor the walking direction of the pedestrians (front/back) influenced the risk assessed considering the two indicators. Given this result, conditions were taken into account indifferently for the analyses that followed. For a given modality of a factor (TTC, lateral offset), all the occurrences have been averaged to get one value by participant per indicator. Then, a one-way 
ANOVA was computed over the 22 resulting values to determine whether the factor has a significant effect on the assessed risk.

The results of the ANOVAs lead to the conclusion of significant effects for the TTC on the two indicators (Risk Max: $F(3,18)=20.74, p<.001$; AUC: $F(3,18)=15.75, p<.001)$. The lateral offset also had an effect although it was only significant for RiskMax (Risk Max: $F(2,19)=3.30, p<.05$; AUC: $F(2,19)=2.60, p<.1)$. In addition, as illustrated on Fig. 5 , the latter the maneuver was triggered, the higher the indicators are. This supports to the intuitive idea that the passenger feels a higher risk when the vehicle starts the avoidance maneuver too late. Likewise, the closer the vehicle was to the pedestrian during the avoidance maneuver, the higher the assessed risk 5 (Fig. 6).

However, post hoc tests of the significant difference of the means (Tukey's HSD test) revealed that the lowest values of both the TTC (2 seconds) and the lateral offset ( 0.5 meter) resulted in a significant increase of the assessed risk.

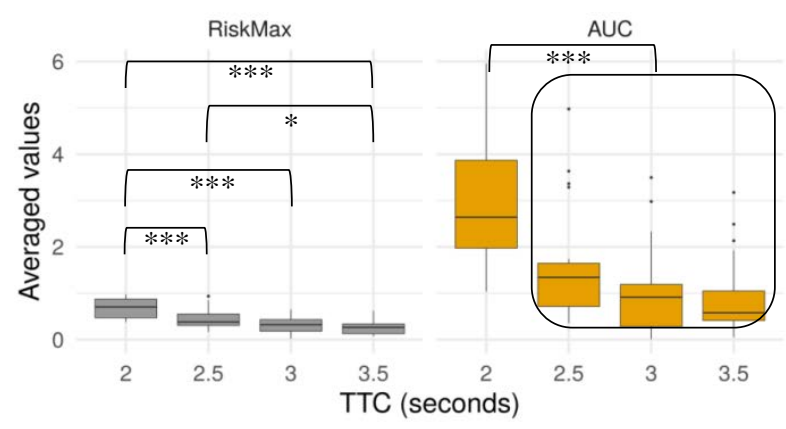

Fig. 5. Effect of the TTC on the two indicators. The stars indicate the results of the post hoc tests $(*: p<.1 ; * *: p<.05 ; * * *: p<.001)$.

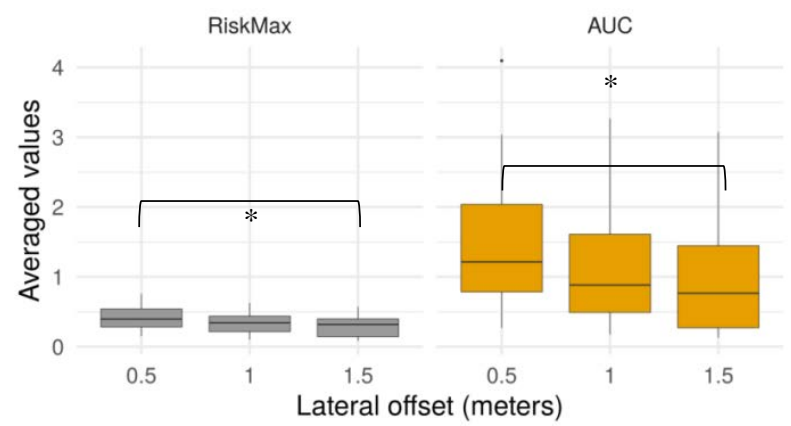

Fig. 6. Effect of the lateral offset on the two indicators. The stars indicate the results of the post hoc tests $(*: p<.1)$. 


\subsection{Risk prediction}

System identification. The data were then used to determine the extent to which the participant's assessment could be modeled as an IR. For this purpose, all avoidance maneuvers were treated independently and optimal coefficients were computed using MATLAB and the System identification toolbox [19]. A set of optimal coefficients corresponds to an IR which fit the best with the observed data in terms of normalized root mean squared error (NRMSE). As shown in the equation 2, NRMSE summarizes the observed differences between observed and predicted data and take into account the actual dispersion of the observed data. Such a performance metric always returns a value lower than 1 , and the nearer the NRMSE is from 1 , the better the predicted values are.

$$
\operatorname{NRMSE}(\text { obs, pred })=1-\sqrt{\frac{\sum_{t}(o b s(t)-\operatorname{pred}(t))^{2}}{\sum_{t}(o b s(t)-\overline{o b s})^{2}}}
$$

Given a moment $t$ :

- $o b s(t)$ : Observed values i.e. the risk assessed by a participant.

- $\operatorname{pred}(t)$ : Predicted value by the ARX model of the risk assessed.

- $\overline{o b s}$ : Mean of the observed values considered in a range of time.

Before estimating the coefficients $\alpha_{i}$ and $\beta_{i}$ (cf. equation 1), the best parameters of the ARX model were obtained $(n a, n b)$. Since no assumption was privileged, all combinations among the grid $n a \in[1 ; 20] \times n b \in[1 ; 20]$ were firstly tested on the data. The performances were compared and finally the values $n a=n b=2$ were retained for all participants. These values were chosen based on an analysis of the performance of all the models, and of the gain observed through the introduction of additional coefficients. Voluntarily, the chosen ARX model was relatively sparse and gave rise to an IR calculated from only 5 coefficients. The fact that $n a=2$ means the autoregressive part of the model concerns only delays of order 1 and 2 . That is, the risk assessed by a participant at a given time $t$ essentially depends on the risk assessed at time $t-1$ and $t-2$. The value of $n b$ is linked to the reaction to exogenous stimulus (retinal expansion rate) and should be interpreted in relation with the value of the last ARX parameter, $n k$. The latter was chosen afterwards by testing all values between 100 milliseconds and 3 seconds (every 50 milliseconds). This parameter was selected for each avoidance maneuver of the participants. As explained before, it corresponds to the number of stimulus samples that occur before seeing a reaction on the assessed risk by the participant. The lower limit $(100 \mathrm{~ms})$ was chosen as the minimum time interval required to perceive a succession of stimuli for a Human [20]. The upper boundary (3s) was selected with regard to data of all participants. Once this parameter was found, the parameter $n b=2$ can be interpreted as the number of stimulus samples that affect the assessed risk after $n k$ time units. More formally, at a given time $t$, the assessed risk depends on the previous values of stimulus which occurred at time $(t-n k)$ and $(t-n k-1)$. 
This procedure of system identification was performed to test whether the risk assessed by a participant could be estimated using an IR and only one exogenous variable. Out of the 704 overtaking maneuvers, only 679 identifications were processed. The remaining 25 maneuvers concerned data with no risk assessed i.e. maneuvers where participants did not perceive risk of collision (cursor remaining at 0). These excluded cases were found randomly among participant and an examination revealed no specific pattern in terms of avoidance conditions or participant profile.

Global model summary. In addition to the system identification two heuristic manipulations were operated.

- The retinal expansion rate exists as soon as the vehicle is approaching to a pedestrian. If the speed of the vehicle remains constant at $30 \mathrm{~km} / \mathrm{h}$, which was the case in the experiment, a pedestrian walking 100 meters in front of the vehicle is already supposed to produce a stimulus for the participant although the risk of collision can reasonably be considered absent. In the ARX model, this causes the IR to be triggered too early and therefore predicts a non-zero assessed risk when no stimulus is actually perceived by participants. To work around this phenomenon, the stimulus series was truncated to keep only values that correspond to TTC below 5 seconds which was the threshold value found based on the data.

- The IR has the disadvantage to keep on estimating a non-zero output (and so a risk predicted different from 0 ) even long after a stimulus occurred. In the context of the experiment, most of participants returned to a value of 0 just after the avoidance maneuver i.e. when the pedestrian was just behind the vehicle. To cope with this phenomenon, the risk predicted by the model was truncated using the distance between the pedestrian and the vehicle: as soon as the vehicle moved away from a pedestrian, the risk predicted was set to 0 .

A global model strategy is then defined and composed of three steps: (step 1) truncate the exogenous stimulus series, (step 2) identify parameters of the ARX model and estimate the coefficients, (step 3) truncate the predicted series.

Performances. Due to the large amount of data expected, performances were compared and judged through median values per participant. The accuracy of the predicted risk estimation was evaluated with the NRMSE and the threshold of 0.6 was chosen to distinguish good and bad fit. The threshold was selected based on the data following observations: performances lower than 0.6 reflected a predicted series which does not fit the observed series (e.g. too large absolute difference between the predicted and the observed values); for performances greater than 0.6 , the predicted series were well correlated and had low absolute differences with the associated observed series. An example of a result with a $N R M S E=0.74$ is illustrated on Fig. 7. The IR (Fig. 7.a) depends on the estimated parameter $n k$ and the system identification results (coefficients $\alpha_{i}$ and $\beta_{i}$ ). This curve trend with a positive skewness is very characteristic and corresponds to the majority of cases. It reflects the fact that the occurrence of a stimulus leads to a rapid increase and then a gradual decrease in the risk assessed. The 
estimated IR is then used in the global model detailed above to compute the predicted series (Fig. 7.b).

Out of the 22 participants under analysis, the median performances of 16 were greater than 0.6. The profiles of the 6 remaining participants have been studied in detail but no specific pattern was found. However, in most cases, the model failed because of the form of the assessed risk. The ARX model assumes that an increase of the stimulus input (retinal expansion rate) should always result in an increase of the output (assessed risk). When this was not the case, the procedure systematically failed to estimate a correct IR. For example, some participants assessed a progressive risk when approaching a pedestrian by staying on the same value for a long time while the rate of retinal expansion increased steadily.

(a)

$$
\text { (a) }
$$

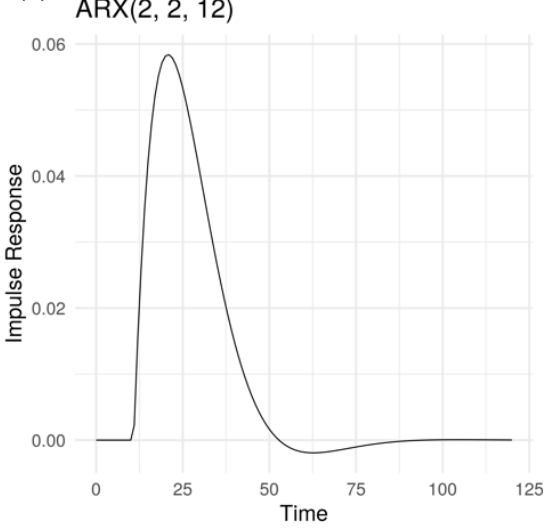

(b)

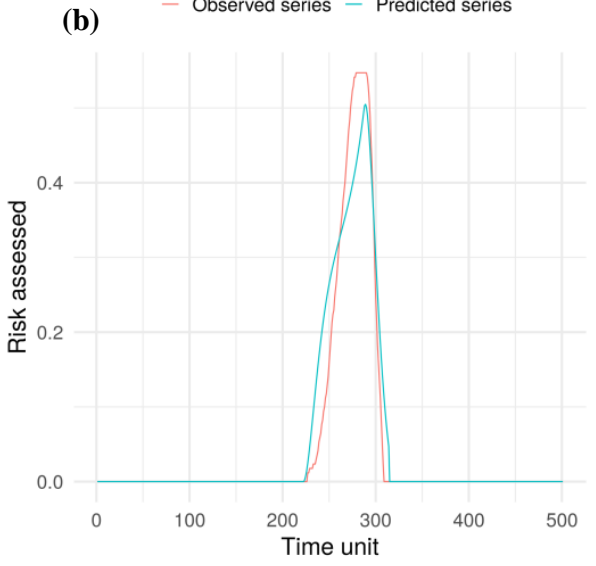

Fig. 7. Result example of the model prediction with the IR (a) obtained after the system identification and the resulting predicted series (b). The NRMSE computed for this example is 0.74 which is judged as satisfying. On the IR, the parameter $n k=12$ can be seen at the beginning of the series that remains at 0 during 12 time units.

As a result, the model strategy was found to be effective for more than $70 \%$ of participants. After this first step, further analysis was conducted to determine whether it was possible to estimate a single IR for all avoidance maneuvers of a given participant. To this end, tests were carried out on all participants and a calculation routine was put in place to progressively aggregate the coefficients. The idea was to simulate the possibility of adjusting future predictions based on the risk assessed during previous avoidance maneuvers for a given participant. For example, the prediction of the assessed risk at the 10th pedestrian avoidance would depend on the observed values for the previous 9 pedestrians. In this example, the coefficients obtained by system identification for the previous 9 maneuvers were averaged. Theoretically, this approach had the advantage of stabilizing the IRs (resulting from the coefficients) as the vehicle progressed. This makes sense because the more the participants experimented with avoidance maneuvers, the more robust the predictive models became. However, this approach yielded disappointing results because the median performance calculated per participant was never higher than that obtained by system identification. Per- 
formance was at least halved for 19 participants; and, surprisingly, for 2 of the remaining 3 participants, the median performance obtained by identification was below the 0.6 threshold mentioned above.

This poor performance can be explained by the fact that the participants' behaviors were not constant during the experiment in terms of the risk assessed by considering only the rate of retinal expansion as an entry stimulus. Therefore, the hypothesis of temporal invariance of IR could not be validated by the data, i.e., the same avoidance maneuver did not produce the same effect on the participant.

\section{Conclusion and discussions}

Results summary. This experiment made it possible to study in real time the risk felt by a passenger when travelling between pedestrians in an autonomous vehicle. Two variables were used to manipulate the vehicle's safety margins: (1) the value of the TTC when the vehicle starts its avoidance maneuver, (2) the lateral safety distance the vehicle leaves with the pedestrian. Two indicators have been proposed to study the influence of these parameters on the assessed risk: the maximum value and the area under the curve. According to these indicators and the results obtained, the TTC at the start of the maneuver and the lateral deviation from the pedestrian have a significant effect on the risk assessed: the lower the TTC or the lateral deviation, the more the participants considered the maneuver to be risky. However, only the extreme values tested for these parameters resulted in significant differences. The risk felt by the participants was also analyzed dynamically for predictive purposes. An autoregressive model with an exogenous variable was tested to predict the risk assessed in real time through the evolution of the rate of retinal expansion when approaching a pedestrian. The results obtained by identifying the parameters resulted in sparse models and satisfactory performance (NRMSE) for more than $70 \%$ of the participants. Nevertheless, attempts to characterize the participants by their impulse response resulted in prediction performances below those obtained by the models with identified parameters.

Conclusion. In view of these results, it is clear that the assessed risk by the participants depends on the dynamics of approach and avoidance of pedestrians by the vehicle. However, the factors tested were not sufficient to explain accurately the assessment behavior observed in the participants. Predicting the risk perceived in an autonomous vehicle probably requires considering more complex independent variables related to the vehicle dynamics. For instance, aggregate the TTC and the relative time separating the vehicle and the obstacle as proposed in [17, 21]. Nevertheless, risk as such remains a concept that is complex to define, and if it is considered to be a feeling (as suggested by Slovic et al. [22]) it would be intuitive and therefore not entirely dependent on objective measures.

It is also possible that the modeling approach considered is not suitable for predicting a risk feeling in a temporal context. For some participants, it was found that assumptions of linearity and temporal invariance were not satisfied. This may reflect a change in the importance or uncertainty that participants placed on the risk of pedes- 
trian collisions (as characterized by Yates and Stones [23]). For future research, a criterion to account for changes in passenger perception of the vehicle behavior (internal model) could therefore be added to the modeling.

\section{References}

1. Reilhac, P., Millett, N., Hottelart, K.: Shifting Paradigms and Conceptual Frameworks for Automated Driving. In: Meyer, G. and Beiker, S. (eds.) Road Vehicle Automation 3. pp. 73-89. Springer International Publishing, Cham (2016)

2. $\quad$ Kyriakidis, M., Winter, J.C.F. de, Stanton, N., Bellet, T., Arem, B. van, Brookhuis, K., Martens, M.H., Bengler, K., Andersson, J., Merat, N., Reed, N., Flament, M., Hagenzieker, M., Happee, R.: A human factors perspective on automated driving. Theor. Issues Ergon. Sci. 20, 223-249 (2019). https://doi.org/10.1080/1463922X.2017.1293187

3. Hollnagel, E.: Time and time again. Theor. Issues Ergon. Sci. 3, 143-158 (2002). https://doi.org/10.1080/14639220210124111

4. Verberne, F.M.F., Ham, J., Midden, C.J.H.: Trust in Smart Systems: Sharing Driving Goals and Giving Information to Increase Trustworthiness and Acceptability of Smart Systems in Cars. Hum. Factors. 54, 799-810 (2012). https://doi.org/10.1177/0018720812443825

5. Basu, C., Yang, Q., Hungerman, D., Singhal, M., Dragan, A.D.: Do You Want Your Autonomous Car To Drive Like You? In: Proceedings of the 2017 ACM/IEEE International Conference on Human-Robot Interaction. pp. 417-425. ACM, New York, NY, USA (2017)

6. Gibson, J.J., Crooks, L.E.: A Theoretical Field-Analysis of Automobile-Driving. Am. J. Psychol. 51, 453 (1938). https://doi.org/10.2307/1416145

7. Elbanhawi, M., Simic, M., Jazar, R.: In the Passenger Seat: Investigating Ride Comfort Measures in Autonomous Cars. IEEE Intell. Transp. Syst. Mag. 7, 4-17 (2015). https://doi.org/10.1109/MITS.2015.2405571

8. Gibson, M., Lee, J., Venkatraman, V., Price, M., Lewis, J., Montgomery, O., Mutlu, B., Domeyer, J., Foley, J.: Situation Awareness, Scenarios, and Secondary Tasks: Measuring Driver Performance and Safety Margins in Highly Automated Vehicles. SAE Int. J. Passeng. Cars - Electron. Electr. Syst. 9, (2016). https://doi.org/10.4271/2016-01-0145

9. $\quad$ Ferrier-Barbut, E., Vaufreydaz, D., David, J.-A., Lussereau, J., Spalanzani, A.: Personal Space of Autonomous Car's Passengers Sitting in the Driver's Seat. In: 2018 IEEE Intelligent Vehicles Symposium (IV). pp. 2022-2029 (2018)

10. SCANeR Studio Release 1.8. AVSimulation, Inc., Boulogne-Billancourt, France

11. Hamilton-Baillie, B.: Towards shared space. URBAN Des. Int. 13, 130-138 (2008). https://doi.org/10.1057/udi.2008.13

12. Moody, S., Melia, S.: Shared space: Research, policy and problems. Proc. Inst. Civ. Eng. - Transp. 167, (2014). https://doi.org/10.1680/tran.12.00047

13. Kaparias, I., Bell, M.G.H., Miri, A., Chan, C., Mount, B.: Analysing the perceptions of pedestrians and drivers to shared space. Transp. Res. Part F Traffic Psychol. Behav. 15, 297-310 (2012). https://doi.org/10.1016/j.trf.2012.02.001

14. Ljung, L.: System Identification: Theory for the User. Pearson Education Canada (1987) 
15. Lee, D.N.: A Theory of Visual Control of Braking Based on Information about Time-toCollision. Perception. 5, 437-459 (1976). https://doi.org/10.1068/p050437

16. Bootsma, R.J., Craig, C.M.: Information Used in Detecting Upcoming Collision. Perception. 32, 525-544 (2003). https://doi.org/10.1068/p3433

17. Lu, G., Cheng, B., Lin, Q., Wang, Y.: Quantitative indicator of homeostatic risk perception in car following. Saf. Sci. 50, 1898-1905 (2012). https://doi.org/10.1016/j.ssci.2012.05.007

18. Chen, R., Sherony, R., Gabler, H.C.: Comparison of Time to Collision and Enhanced Time to Collision at Brake Application during Normal Driving. SAE International, Warrendale, PA (2016)

19. MATLAB and the System Identification Toolbox Release 2018b. The MathWorks, Inc., Natick, Massachusetts, United States

20. Lieury, A.: Psychologie cognitive. Dunod (2015)

21. Kondoh, T., Yamamura, T., Kitazaki, S., Kuge, N., Boer, E.R.: Identification of Visual Cues and Quantification of Drivers' Perception of Proximity Risk to the Lead Vehicle in Car-Following Situations. J. Mech. Syst. Transp. Logist. 1, 170-180 (2008). https://doi.org/10.1299/jmtl.1.170

22. Slovic, P., Finucane, M.L., Peters, E., MacGregor, D.G.: Risk as analysis and risk as feelings: Some thoughts about affect, reason, risk, and rationality. Risk Anal. 24, 311322 (2004)

23. Yates, J.F., Stone, E.R.: The risk construct. In: Risk-taking behavior. pp. 1-25. John Wiley \& Sons, Oxford, England (1992) 